\title{
Efficience des stratégies endogènes d'adaptation du secteur agricole aux changements climatiques dans le bassin de la région de Thiès (Sénégal)
}

\author{
Souleymane DIALLO ${ }^{*}$, Hassan Bismarck NACRO ${ }^{2}$ et Aminata N'DIAYE ${ }^{1}$ \\ ${ }^{1}$ Université Cheikh Anta Diop de Dakar, Faculté des Lettres et Sciences Humaines, Département de \\ Géographie, Dakar, Sénégal, BP : 5005 Dakar Fann-Résidence, Dakar, Sénégal. \\ ${ }^{2}$ Université Polytechnique de Bobo-Dioulasso, Laboratoire d'Etude et de Recherche sur la Fertilité du Sol, \\ Burkina Faso, BP : 1091 Bobo 01, Burkina Faso. \\ *Auteur correspondant ; E-mail : timsouley@gmail.com
}

\section{REMERCIEMENTS}

Nous exprimons notre profonde gratitude envers le projet INTRA ACP HAAGRIM coordonné par l'Université Gaston Berger de Saint-Louis pour son apport financier qui a été très significative pour la réalisation de ce travail.

\section{RESUME}

Les difficultés du secteur agricole causées par les changements climatiques et l'activité anthropique ne sont pas restées sans réponse dans le monde. Des mesures d'atténuation d'émission des gaz à effet de serre ont été prises à grande échelle auxquelles viennent s'adjoindre des stratégies d'adaptation. Selon le Groupe Intergouvernemental pour l'évolution du climat (GIEC, 2007), l'amélioration des pratiques agricoles (gestion du bétail et des effluents agricoles, de la fertilisation azotée ...) peut contribuer de manière significative à réduire les émissions et à augmenter le contenu en carbone et la capacité de puits des sols. Dans le bassin de la région de Thiès (Sénégal), plusieurs mesures d'adaptation ont été prises et mises en œuvre. L'objectif de cette étude est de voir si ces stratégies de lutte mises en œuvre ont porté leurs fruits. La méthodologie repose sur la documentation des informations déjà disponibles sur le thème et portant sur la zone de recherche, sur les enquêtes de terrain et sur les entretiens avec les différents acteurs concernés, enfin sur l'analyse des données climatiques de l'Agence Nationale de l'Aviation Civile et de la Météorologie (ANACIM) et des données agronomiques de la Direction de l'Analyse, de la Prévision et des Statistiques Agricoles (DAPSA). Les résultats montrent que l'essentiel des solutions mises en place ont été rentables.

(C) 2017 International Formulae Group. All rights reserved.

Mots clés : Stratégie endogène, adaptation, secteur agricole, changement climatique, bassin de Thiès, Sénégal.

\section{Efficiency of endogenous adaptation strategies of the agricultural sector to climate change in the Thies region basin (Senegal)}

\section{ABSTRACT}

The difficulties of the agricultural sector caused by climate change and human activity have not been unanswered in the world. Measures to mitigate greenhouse gas emissions have been taken on a large scale, with adaptation strategies being added. According to the Intergovernmental Panel on Climate Change (IPCC, 2007), improving agricultural practices (management of livestock and agricultural effluents, nitrogen fertilization, 
etc.) can significantly contribute to reducing emissions and increasing carbon content and soil sink capacity. In the basin of the region of Thies (Senegal), several adaptation measures have been taken and implemented. The aim of this study is to see whether these control strategies have been successful. The methodology is based on the documentation of the information already available on the topic, covering the research area, field surveys and interviews with the various stakeholders, and finally the analysis of climate data from the National Agency Civil Aviation and Meteorology (ANACIM) and agronomic data from the Directorate of Agricultural Analysis, Forecasting and Statistics (DAPSA). The results show that most of the solutions put in place have been profitable.

(C) 2017 International Formulae Group. All rights reserved.

Keywords: Endogenous strategy, adaptation, agricultural sector, climate change, Thies basin, Senegal.

\section{INTRODUCTION}

La crise qui frappe l'activité agricole dans le bassin de la région de Thiès a des origines multiples. Les récentes enquêtes de terrain (Diallo, 2012) ont montré que l'agriculture est une activité dont la réussite implique une connaissance et une maîtrise des conditions climatiques, une gestion rationnelle de la ressource terre, une dotation aux exploitants agricoles d'intrants, de matériels, etc. Au-delà, les difficultés que les autres secteurs économiques subissent doivent -être intégrées dans les réflexions tendant vers le redressement et l'épanouissement de l'activité agricole car elles affectent l'agriculture même si c'est indirectement. Le secteur agricole sera plus productif si les facteurs (semences, intrants agricoles, matériel agricole, crédit agricole, etc...) contraignants le développement de la pratique agricole, exerçaient plus d'effets menant vers l'avant l'activité (ANSD, 2010). Face à cela, des réponses ont été apportées et ont eu des impacts notoires. Il convient aussi de souligner que beaucoup des stratégies d'adaptations mises en place au Sénégal et dans le bassin de Thiès en particulier n'ont pas atteint leur objectif et se sont soldées parfois par des échecs. L'objectif ici est de voir si les démarches utilisées ou les initiatives élaborées et concrétisées dans le bassin de la région de Thiès afin de promouvoir l'activité agricole par les ménages agricoles et les acteurs impliqués sont rentables. Ainsi, suite à cela, l'efficience des méthodes peuvent-être évaluées.

\section{MATERIEL ET METHODES}

Présentation de la zone d'étude

La zone d'étude se localise à l'Ouest du Sénégal entre les latitudes $14^{\circ} 02^{\prime}$ et $15^{\circ}$ $27^{\prime}$ Nord et les longitudes $16^{\circ} 09^{\prime}$ et $17^{\circ} 12^{\prime}$ Ouest. Elle couvre une superficie de $3525 \mathrm{~km}^{2}$ et est limitée au Nord par la région de Louga, au Sud par la région de Fatick, à l'Est par les régions de Diourbel et de Fatick et à l'Ouest par la région de Dakar et l'Océan Atlantique. Elle comprend 3 Départements: le Département de Thiès, le Département de Mbour et le Département de Tivaouane. Le climat du bassin de la région de Thiès est influencé par des courants marins. En effet, le bassin de la région de Thiès se situe dans une zone de transition soumise à l'influence des alizés maritimes et de l'harmattan, elle présente un climat de type soudano-sahélien: Sud, Sud-est et plus sahélien au Nord et Nordest. La zone Ouest quant à elle présente un climat Sub-canarien (ANSD, 2010). Le relief y est relativement plat excepté certaines zones faiblement accidentées. Les sols, les ressources hydriques et la végétation subissent de fortes dégradations causées par les modifications climatiques mais aussi par la pression anthropique exacerbée. La région de Thiès est parmi les plus peuplées du Sénégal avec une population de 1788864 habitants et qui possède de loin le plus de ménages agricoles avec un taux de $12,8 \%$; ce qui montre la place primordiale de l'agriculture. Toutefois, les autres secteurs d'activités y sont développés et concurrencent l'agriculture. 


\section{Collecte des données}

Les informations et les données utilisées dans le cadre de cette étude proviennent de la revue littéraire des documents disponibles et traitants de cet aspect. La plupart de ces documents sont numériques. Ils sont obtenus par l'intermédiaire des collègues universitaires et des collègues du secteur professionnel mais aussi via des bibliothèques numériques telles que la bibliothèque universitaire de l'Université Cheikh Anta Diop (UCAD), la bibliothèque du Conseil Africain et Malgache pour l'Enseignement Supérieur (CAMES) et des revues. Les documents papiers consultés dans ce cadre sont le plus souvent des documents lus au moment de la préparation d'un Mémoire de Master II qui a porté sur l'ancienne Communauté Rurale de Fandène située dans le bassin de la région de Thiès. Puis, les informations sur les techniques de résilience développées et leur efficience ont été complétées par les données de terrain et les informations issues des entretiens. Les ultimes données utilisées dans le cadre de cette étude proviennent de l'Agence Nationale de l'Aviation Civil et de Météorologie (données climatiques) et de la Direction de l'Analyse, de la Prévision et des Statistiques Agricoles (données agronomiques). Elles sont constituées de données pluviométriques et de données agronomiques sur une période de 17 ans (1996-2013).

\section{Méthodologie}

Le questionnaire élaboré portant sur les stratégies de résistance des populations face aux difficultés dont sont confrontées le secteur agricole a été soumis à un nombre de 383 ménages agricoles sur un total de 187085 ménages que compte le bassin de la région de Thiès. L'échantillon de l'étude a été choisi sur la base de la formule de Bernoulli en acceptant une marge d'erreur de $10 \%$.

La formule permettant de calculer la taille de l'échantillon est la suivante : $\mathrm{n}=(1,96)^{2 *} \mathrm{~N} /(1,96)^{2}+\mathbf{l}^{2 *}(\mathrm{~N}-1)$

Où avec un niveau de confiance : $95 \%=1,96$

$\mathbf{n}=$ taille de l'échantillon à interroger,

$\mathbf{N}=$ taille de l'univers investigué (l'ensemble des ménages agricoles du bassin la région de Thiès 187085 ménages)

$\mathbf{I}=$ largeur de la fourchette exprimant la marge d'erreur (10\%)

Le focus group a aussi été un moyen employé sur le terrain pour atteindre les objectifs de l'enquête pourvu que l'échantillonnage qu'on s'est fixé est élevé et le nombre de questions posé aux enquêtés est important.

Deux niveaux sont utilisés pour l'analyse des données d'enquête et des données climatiques. Il s'agit de l'analyse descriptive univariée et l'analyse descriptive bivariée.

L'analyse descriptive univariée consiste à étudier successivement les caractères individuels de chaque variable. Selon la nature des variables que nous avons étudiées (qualitative nominale, ou quantitative numérique), différents types d'analyses sont mis en œuvre.

Pour les variables qualitatives ou nominales, l'analyse est faite en utilisant les tableaux de fréquences, les tableaux croisés et des graphiques

Pour les variables quantitatives discrètes ou continues, les analyses effectuées sont l'analyse des tendances centrales (la moyenne), l'analyse des dispersions (le minimum, le maximum, l'écartype, le coefficient de variation et les percentiles).

Les analyses descriptives bivariées ont permis de traiter simultanément deux variables afin d'évaluer la relation qui existe entre les deux questions.

La série de données climatiques a été scindée en deux phases : la phase sèche et la phase humide (années sèches et années humides) et les rendements agricoles annuels des différentes spéculations ont été analysés en fonction de ce classement. 
L'indice pluviométrique de Lamb et Peppler (1992, cité par Owusu et Klutse, 2013) a été utilisé pour scinder les années de la série.

\section{-Indice pluviométrique de Lamb :}

$$
I=\frac{P_{i}-\sigma}{\bar{P}}
$$

$I=$ : Indice pluviométrique de Lamb; $P_{i}$ :

Pluviométrie de l'année i, $\bar{P}$ : Pluviométrie moyenne interannuelle sur la période de référence, $\sigma$ : Écart type de la pluviométrie interannuelle sur la période de référence.

Le bilan hydrique a été calculé pour estimer le potentiel hydrique du sol (la réserve hydrique du sol) afin de savoir si l'humidité du sol peut maintenir les plantes en vie pendant les périodes de long stress hydrique.

\section{Bilan hydrique $=$ Bilan hydrique jour $-1+$ Pluie-Evaporation}

La méthode de Penman Monteith qui permet d'estimer l'évapotranspiration des plantes figure également parmi les méthodes dont on a eu à recourir dans le cadre de cette étude. Elle permet d'estimer comme déjà mentionné l'évapotranspiration des plantes ou les pertes d'eaux par transpiration des plantes. Elle demande au préalable l'acquisition des différents éléments :

1. La pluie moyenne mensuelle ;

2. Les températures maximales et minimales mensuelles ;

3. L'humidité relative moyenne mensuelle ;

4. La vitesse moyenne mensuelle du vent ;

5. L'insolation moyenne mensuelle ;

6. Les coordonnées géographiques et l'altitude du site de l'étude.

\section{Logiciels utilisés}

SPHINX a servi à l'élaboration du questionnaire, SPSS pour le traitement des réponses, INSTAT+ et CROPWAT pour la détermination des variables statistiques et EXCEL pour les tableaux et graphiques.

\section{RESULTATS}

L'efficacité des types de fertilisant et techniques de conservation de la fertilité des sols

Les types de fertilisants et les techniques de conservation de la fertilité des sols sont entre autres la fumure, le compost, les engrais minéraux, la jachère, le pacage des animaux aux champs, le brulis, etc. La fumure, citée par $61,1 \%$ des ménages agricoles, est de loin devant les engrais et le compost, le fertilisant le plus utilisé pour remédier à la carence en minéraux des sols. La jachère est la technique la plus sollicitée pour la conservation de la fertilité des sols dans le bassin de la région de Thiès avec $8,4 \%$ des exploitants agricoles qui l'adoptent (Enquête de terrain, 2015). Plus de 56,7\% des producteurs locaux affirment être satisfaits de l'usage de ces fertilisants et techniques de conservation des sols et $12,3 \%$ affirment être très satisfaits (Tableau 1). Ce qui montre l'efficience de ces pratiques de maintien de la fertilité dans les terres de culture du bassin de la région de Thiès.

\section{Efficacité des méthodes de lutte contre l'érosion}

Les études de terrain ont révélé que l'érosion des sols est un problème qui touche une masse importante des exploitants agricoles du bassin avec plus de $81 \%$ qui avouent être victimes de ce phénomène. Les solutions de lutte sont entre autres les haies vives, l'agroforesterie, le système de culture qui bloque le ruissellement de l'eau (érosion hydrique), etc. Les résultats issus de ces méthodes de lutte contre l'érosion s'avèrent peu efficaces pour une grande partie de ces exploitants puisque plus de $51,9 \%$ d'entre eux ne sont pas satisfaits des effets de ces méthodes. Cependant, $47,1 \%$ des ménages agricoles trouvent une satisfaction dans 
l'emploi de ces moyens de lutte et $1 \%$ sont très satisfaits (Tableau 1). Globalement, nous pouvons dire que ces techniques sont fructueuses car $48,1 \%$ des exploitants agricoles $\mathrm{du}$ bassin trouvent ces techniques efficaces.

Efficience des mesures de lutte contre l'effet du stress hydrique sur l'agriculture dans le bassin

Les solutions adoptées par les exploitants agricoles restent les mêmes car selon eux les mécanismes permettant un approvisionnement en eau suffisante pour l'agriculture ne sont pas concrétisés par les autorités locales (la Mairie, le Service Régional de l'Assainissement, la Division régionale de l'Hydraulique, etc.) et leurs partenaires (les Organisations Non Gouvernementales, la Société Des Eaux, etc.). A cet effet, 74,4\% des ménages agricoles disent qu'il n'y a pas de soutien en la matière venant des autorités (Enquête de terrain, 2015). Ainsi, les solutions qu'ils ont adopté pour faire face à cela restent les mêmes (approvisionnement via robinets, puits, forages pour certains exploitants agricoles, etc.). Toutefois, l'accès à ces ressources est sursaturé. Ainsi, les résultats sur la satisfaction en eau sont insuffisants car $71,6 \%$ des producteurs locaux sont insatisfaits de se contenter de ces seuls moyens disponibles pour leur approvisionnement en eau et seuls 28,4\% affirment être satisfaits (Tableau 1); ce qui est faible.

Diversification des pratiques agricoles dans le bassin de la région de Thiès

La pratique conjointe de type de culture différent dans le bassin

L'association culturale demeure parmi les stratégies de lutte rencontrée lors des investigations sur le terrain (Figure 1). Dans certaines localités du bassin où les conditions nécessaires pour la pratique agricole sont réunies, les producteurs locaux ont opté pour cette méthode afin d'accroître leurs productions agricoles et de réduire la paupérisation à laquelle ils sont confrontés.

Les enquêtes de terrain montrent que les ménages agricoles qui s'activent dans plusieurs types de pratiques agricoles sont satisfaits de la juxtaposition de cette diversité culturale avec un taux de $54,6 \%$ et $1 \%$ trouvent une forte satisfaction après diversification de leur pratique agricole. En revanche, $44,4 \%$ ne sont pas satisfaits; ce qui constitue une portion importante mais tout de même inférieure à ceux qui y trouvent satisfaction. Il en découle que cette diversité de pratique agricole est une méthode efficace pour le redressement des productions agricoles (Tableau 1).

Etude de quelques spéculations (variétés agricoles) en résilience à la variabilité climatique

Variabilité pluviométrique et résistance des cultures

L'étude s'appuie sur les données agronomiques de la DAPSA de 1996 à 2013 et les données pluviométriques de l'ANACIM sur la même période. Une étude de l'évolution des chroniques pluviométriques dégage deux périodes : une période sèche allant de 1996 à 2006 et une période humide de 2007 à 2013. Cependant, la méthode de Lamb a permis de distinguer les années sèches de la série des années humides (Figure 2).

La résilience des spéculations agricoles à la variabilité climatique peut-être observée dans l'évolution des rendements agricoles annuels comme le montre le Tableau 2.

L'observation du Tableau 2 montre qu'il n'existe pas une nette différence entre les rendements des années humides et les rendements des années sèches pour ces différentes spéculations les plus cultivées dans le bassin de la région de Thiès. Le maximum de rendement du mil pendant les années humides de la série est de $2188 \mathrm{~kg} / \mathrm{ha}$ en 2012 et le maximum des années humides est de 
$1623 \mathrm{~kg} / \mathrm{ha}$ en 2006 soit une différence de 565 $\mathrm{kg} / \mathrm{ha}$ et le minimum des années sèches $(1096$ $\mathrm{kg} / \mathrm{ha}$ ) est enregistré en 1997 et le minimum des années humides $(737 \mathrm{~kg} / \mathrm{ha})$ est observé en 2007. S'agissant de l'arachide, du sorgho et du manioc, de forts rendements sont observés pendant les années sèches avec respectivement des maximums de $1734 \mathrm{~kg} / \mathrm{ha}$ en $2006,1511 \mathrm{~kg} / \mathrm{ha}$ en 2006 et $62000 \mathrm{~kg} / \mathrm{ha}$ en 1997. Les études de terrain en sont une confirmation. Ces études montrent que $74,3 \%$ des ménages agricoles sont satisfaits des variétés agricoles qu'ils utilisent dans leurs exploitations agricoles, $3,5 \%$ sont très satisfaits de ces variétés et alors que $22,2 \%$ des ménages agricoles ne le sont pas sur les 230 fermiers agricoles qui disent semer dans leurs champs de nouvelles variétés de culture productive (Tableau 3).

\section{Effets des bonnes conditions climatiques} récentes sur les rendements agricoles

Toutefois, ces rendements satisfaisants des exploitations agricoles ne viennent pas exclusivement des types de variétés utilisés, une analyse minutieuse du climat montre que durant les dernières années, les conditions climatiques sont devenues favorables à de bonnes saisons agricoles (Tableau 4).

Les statistiques descriptives des deux phases de la série montre que les cumuls de pluie annuels ont augmenté, les dates de début de la pluie ont reculé, les dates de fin ont avancé, le nombre de jours de pluie et la longueur des saisons pluvieuses ont aussi augmenté. Mais aussi, l'observation de l'évolution temporelle de la pluie (élément le plus déterminant dans les rendements agricoles annuels des exploitants) montre des pluies harmonisées durant les dernières années.

La tendance mensuelle des épisodes sans pluie dans le bassin de la région de Thiès est illustré par le Tableau 5 (moyenne des épisodes sans pluie de juin à octobre série 1996-2013) qui montre l'importance de la longueur de la moyenne des pauses de la série au mois de juin (158 jours qui s'explique par l'absence de pluies notée au courant de ce mois) et une baisse de la longueur de ces dernières du mois de juin au mois de septembre de 158 jours à 6,9 jours. Ces séquences augmentent en durée au mois d'octobre avec une moyenne de 17,6 jours. L'observation de ce tableau montre que les stress hydriques apparaissant durant les mois de juin et de juillet dans le bassin de la région de Thiès nuisent aux plantes car en ce moment, la réserve hydrique n'est pas encore constituée, les bilans hydriques sont nuls à cette période (Tableau 5).

Aussi, on observe même une baisse des longues pauses du mois de juin et de juillet dans la phase humide et en principe une baisse récente (durant les dernières années) puisque la phase humide concerne plus les dernières années de la série (1996-2013) mis à part les années 2002, 2003, 2004 et 2006. Ces épisodes sans pluie qui atteignaient des longueurs de 33 jours (juin-juillet: les pluies reçue au 3 juillet $0,1 \mathrm{~mm}$ et 8 juillet $0,3 \mathrm{~mm}$ sont $\leq 1 \mathrm{~mm}$ ) en 1996, 28 jours (juin-juillet) en 1997 et 16 jours (juillet) en 2002 se sont rétrécis en durée pendant la phase humide. Les pauses les plus longues durant la phase humide sont de 16 jours (juin-juillet) en 2008, 14 jours (juin-juillet) en 1999 et 12 jours (juillet) en 2013.

L'étude mensuelle de ces épisodes secs montre aussi que le mois de juin et de juillet de la phase sèche a des séquences plus longues que celles des mois de juin et de juillet de la phase humide avec des maximums de 21 jours et de 18 jours respectivement juin et juillet (phase sèche) contre 14 jours et 12 jours juin et juillet (phase humide). S'agissant de la durée des pauses pendant les mois d'août et de septembre, c'est plutôt l'inverse qui se produit. Ils sont plus longues pendant la phase humide avec un maximum de 9 jours (septembre) en 1999, 8 jours (août) en 2007 et 8 jours (septembre) en 2008 alors que pendant la phase sèche de la série, ils n'atteignent les 8 jours qu'en 2002 et en 2004 respectivement au mois de septembre et au mois d'août. Ces épisodes sont le plus souvent inférieurs ou égaux à 5 jours pendant la phase sèche. Pour 
le mois d'octobre, nous notons des pauses plus longues pendant la phase humide de la série avec une moyenne de 11,6 jours contre une moyenne de 10,8 jours pendant la phase sèche.

Les conclusions qui découlent de cette étude des séquences sèches sont entre autres pendant la phase sèche. Le nombre de jours de pluies est très élevé pendant les mois d'août et de septembre et par conséquent les pauses sont plus élevés pendant les mois de juin et de juillet. Le paradoxe est observé pendant les dernières années, là le nombre de jours de pluies s'est accru pendant les mois de juin et de juillet et les pauses ont augmenté en durée pendant les mois de septembre et d'août; c'est surtout la fréquence de ces longues séquences qui a augmenté. Ce qui nous permet de dire que la pluie pendant la phase d'installation des cultures (juillet) a récemment accrut pendant la phase humide.

L'étude des moyennes mensuelles de chaque série montre une forte hausse de la pluie du mois de juillet allant d'une moyenne de $61,1 \mathrm{~mm}$ (phase sèche) à 107,9 $\mathrm{mm}$ (phase humide) et ce qui rend moins agressif l'effet des séquences sèches sur les cultures et favorise des rendements satisfaisants puisque la réserve hydrique se constitue précocement au mois de juillet avec les fortes pluies de ce mois.

Concernant les mois d'août et de septembre, la moyenne a largement augmenté de la phase sèche à la phase humide. Cette moyenne est passée de 142,3 $\mathrm{mm}$ (phase sèche) à 252,8 $\mathrm{mm}$ (phase humide) au mois d'août. Tandis que la hausse de la moyenne du mois de septembre entre les deux phases est moins significative de $119,7 \mathrm{~mm}$ (phase sèche), elle est à 158,3 mm. Ce qui accroît davantage la réserve hydrique du sol (les dernières années) et rend nulle l'action des épisodes secs sur les cultures mais aussi peut même servir d'apport hydrique aux cultures durant les mois de juin et juillet de la saison suivante (Figure 3). En effet, plus les réserves hydriques du sol sont importantes, plus la durée d'approvisionnement des plantes est importante. La moyenne du mois d'octobre a aussi suivi la même tendance de $27,8 \mathrm{~mm}$ pendant la phase sèche, elle est à $52,7 \mathrm{~mm}$ pendant la phase humide. Ce qui amplifie la résistance des cultures aux épisodes secs de ce mois.

Si l'on observe la fréquence des épisodes sans pluie, on voit qu'elles sont plus récurrentes pendant les saisons pluvieuses de la phase humide comme le montre le Tableau 6. Il y a donc une hausse de ces épisodes pendant la phase humide. Cependant, la hausse des pluies du mois de juillet, août, septembre et octobre les rend moins menaçant que ceux de la phase sèche. Une étude mensuelle montre que la hausse de ces épisodes est accentuée pendant les mois de juillet et d'août et que le mois de juin est marqué par une baisse.

La conclusion finale qui découle de l'analyse de la résistance des variétés agricoles aux conditions climatiques montre que c'est plutôt le climat qui se régularise avec une bonne répartition de la pluie dans le temps, un début plutôt que celui d'avant, une fin tardive comparée à celui d'avant, une plus longue saison pluvieuse, une hausse du nombre de jours de pluies mais aussi une hausse de la quantité de pluie et tout ceci explique les bons rendements agricoles enregistrés au courant des dernières années. Le climat a réuni les conditions nécessaires pour de bons rendements agricoles.

\section{Efficience des techniques de culture}

Les techniques de culture répertoriées sur le terrain sont entre autres la culture continue, la rotation céréale/légumineuse et l'association culturale, Dans le bassin de la région de Thiès, la plus grande partie des ménages agricoles soutiennent développer dans l'exercice de leur activité des techniques menant à la minimisation des pertes de production. Un peu plus de $65,3 \%$ des exploitants agricoles disent pratiquer la technique de culture prolifératrice de production élevée et une masse moins importante n'ont pas adopté ces techniques 
(Enquête de terrain, 2015). La proportion de ménages agricoles qui ne s'adonnent pas à ces techniques de culture plus productives, sont des producteurs locaux qui sont attachés à la monoculture (culture continue).

La rotation culturale et l'association culturale sont les deux principaux types de pratique agricole qui produisent le plus et qui même en cas de perturbations permettent aux exploitants de minimiser les pertes. Les systèmes de culture développés pour contrecarrer les productions agricoles dans les exploitations semblent donner des résultats efficients aux producteurs locaux du bassin de la région de Thiès. Les statistiques montrent que plus de $74,4 \%$ des interrogés sont satisfaits de l'applicabilité de ces techniques et $4,8 \%$ disent que ces techniques portent une très grande satisfaction à leur endroit. Evidemment, le taux qui ne trouve pas gain de satisfaction sur l'utilisation de ces systèmes de culture dans leur exploitation est moindre avec $20,8 \%$ des exploitants (Tableau 7).

Tableau 1 : Perception de l'efficacité des moyens de lutte mises en place par les exploitants (Enquête de terrain, 2015).

\begin{tabular}{lcccccccc}
\hline & $\begin{array}{c}\text { Types de fertilisant } \\
\text { et technique de } \\
\text { conservation }\end{array}$ & $\begin{array}{c}\text { Stress } \\
\text { hydrique }\end{array}$ & \multicolumn{2}{c}{$\begin{array}{c}\text { Diversification des } \\
\text { pratiques agricoles }\end{array}$} & & \\
\cline { 2 - 7 } & Effectif & $\%$ & Effectif & $\%$ & Effectif & $\%$ & Effectif & $\%$ \\
\hline Insatisfait & 119 & 31 & 167,5 & 71,6 & 167 & 44,4 & 160 & 51,9 \\
\hline Satisfait & 217 & 56,7 & 66,5 & 28,4 & 205 & 54,5 & 145 & 47,1 \\
\hline Très satisfait & 47 & 12,3 & & 0 & 4 & 1,1 & 3 & 1 \\
\hline Total & 383 & 100 & 234 & 100 & 376 & 98,2 & 308 & 80,4 \\
\hline $\begin{array}{l}\text { Système } \\
\text { manquant }\end{array}$ & & 0 & 149 & 38,9 & 7 & 1,8 & 75 & 19,6 \\
\hline
\end{tabular}

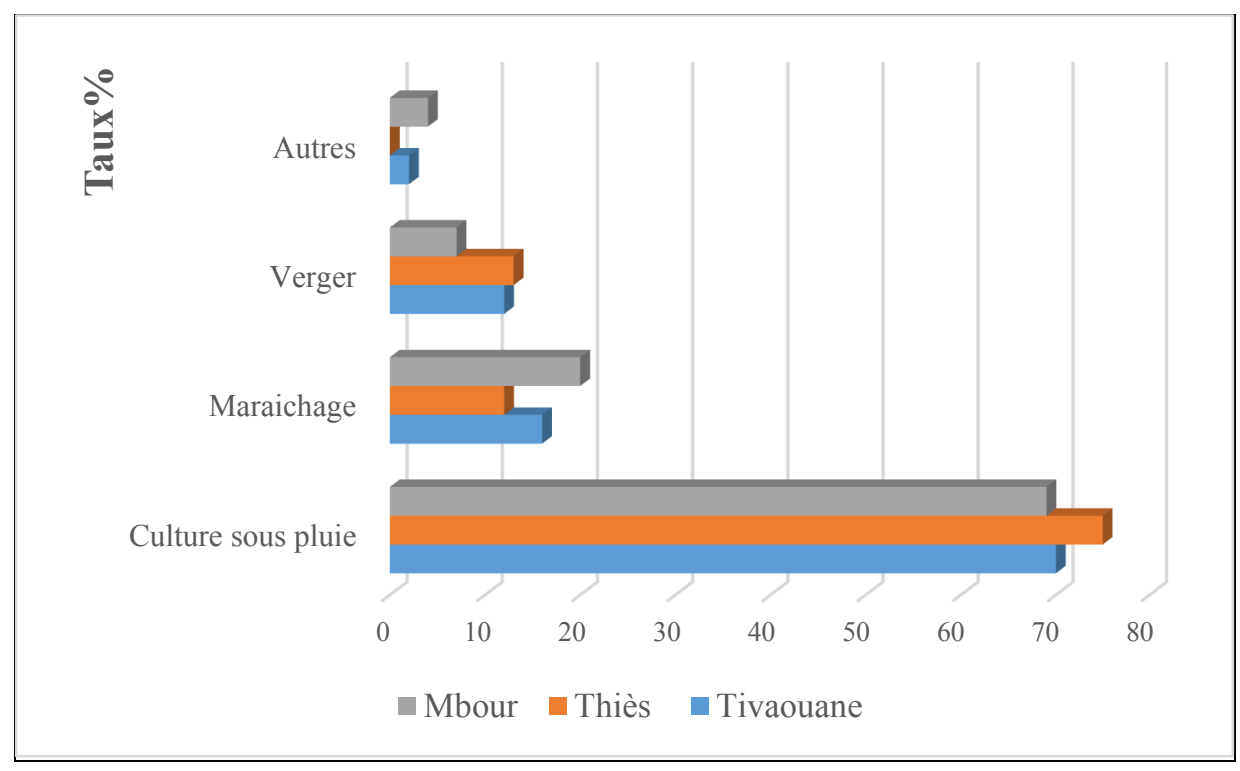

Figure 1: Proportion des exploitants agricoles dans chaque type d'activité agricole par Département (Enquête de terrain, 2015). 


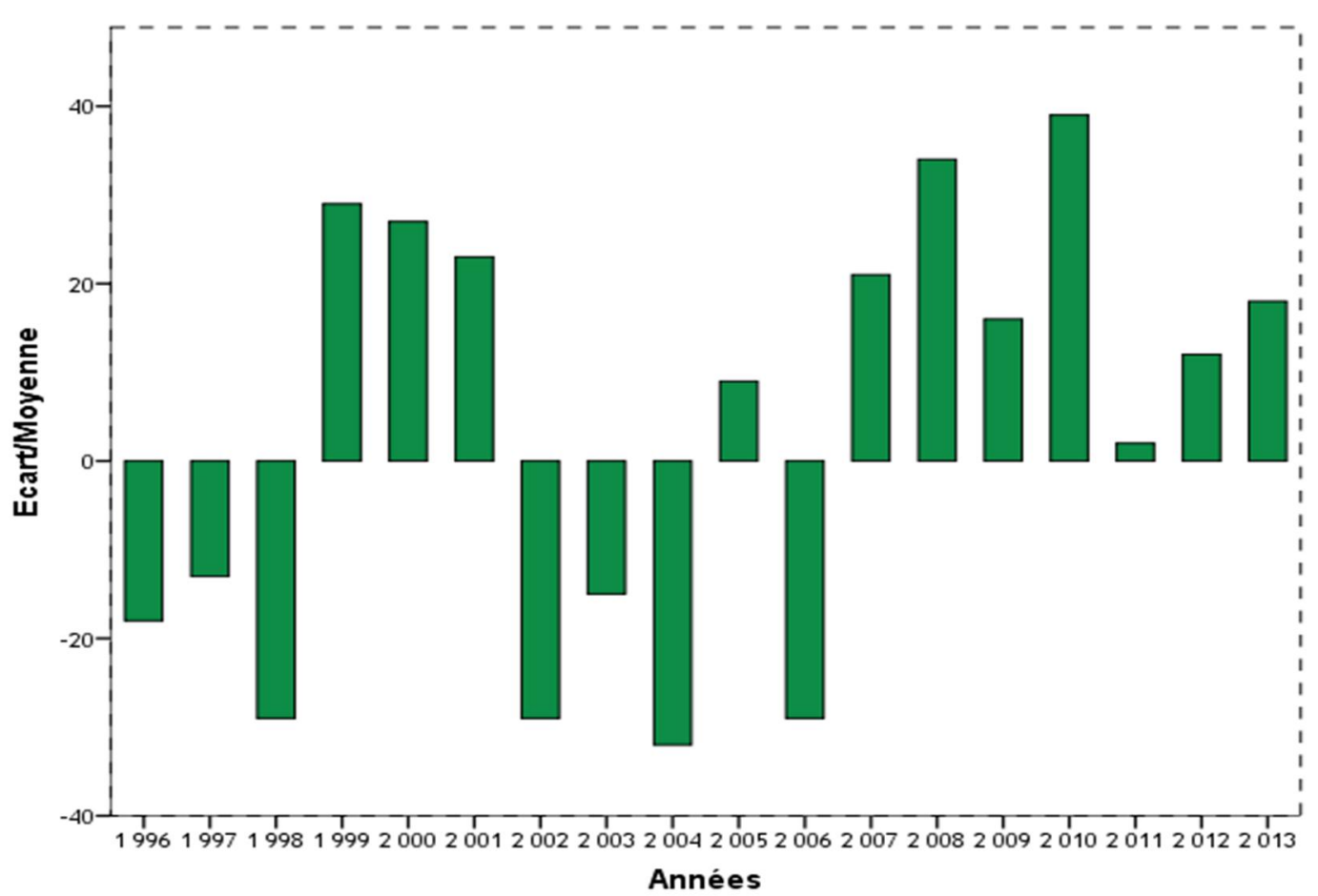

Figure 2 : Ecart à la moyenne de la pluviométrie dans le bassin de la région de Thiès de 1996 à 2013 (ANACIM, 2013).

Tableau 2 : Comparaison des rendements $\mathrm{kg} / \mathrm{ha}$ de quelques spéculations agricoles entre certaines années sèches et humides de la série (DAPSA, 2014).

\begin{tabular}{ccccc}
\hline Années humides & Arachide Huilerie & Mil & Sorgho & Manioc \\
\hline $\mathbf{2 0 0 7}$ & 1.089 & 737 & 1.009 & 15.000 \\
$\mathbf{2 0 0 8}$ & 1.751 & 2.127 & 2.415 & 26.000 \\
$\mathbf{2 0 0 9}$ & 2.722 & 2.058 & 1.237 & 26.000 \\
$\mathbf{2 0 1 0}$ & 2.927 & 2.143 & 2.463 & 24.500 \\
$\mathbf{2 0 1 1}$ & 1.889 & 1.753 & 2.119 & 24.500 \\
$\mathbf{2 0 1 2}$ & 3.052 & 2.188 & 2.205 & 25.500 \\
$\mathbf{2 0 1 3}$ & 2.015 & 1.373 & 1.955 & 23.000 \\
Années sèches & Arachide Huilerie & Mil & Sorgho & Manioc \\
$\mathbf{1 9 9 7}$ & 1.098 & 1.096 & 550 & 62.000 \\
$\mathbf{1 9 9 8}$ & 1.656 & 1.108 & 1.391 & 15.000 \\
$\mathbf{2 0 0 3}$ & 1.120 & 1.276 & 1.143 & 15.000 \\
$\mathbf{2 0 0 6}$ & 1.734 & 1.623 & 1.511 & 22.500 \\
\hline
\end{tabular}


Tableau 3 : Rentabilité des variétés agricoles face à la variabilité pluviométrique.

\begin{tabular}{lcc}
\hline & \multicolumn{2}{c}{ Variabilité pluviométrique et résistance des cultures } \\
\cline { 2 - 3 } & Effectif & $\mathbf{\%}$ \\
\hline Insatisfait & 51 & 22,2 \\
Satisfait & 171 & 74,3 \\
Très satisfait & 8 & 3,5 \\
Total & 230 & 60,1 \\
Système manquant & 153 & 39,9 \\
\hline
\end{tabular}

Tableau 4 : Statistiques descriptives des paramètres clés de la pluviométrie de la série 1996-2013 (ANACIM, 2013).

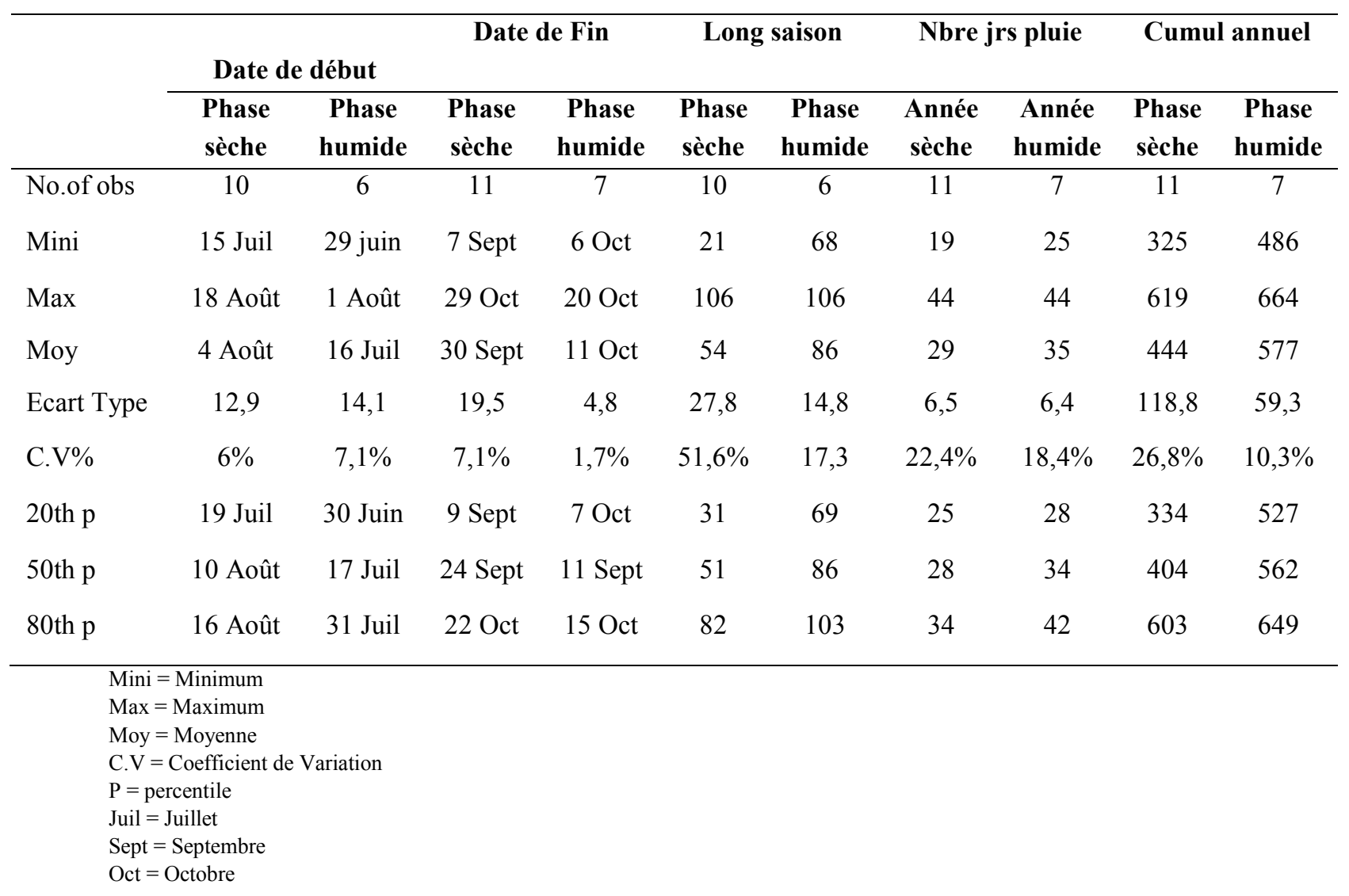


Tableau 5 : Etude de l'impact des périodes sans pluie sur les rendements agricoles des cultures du bassin de la région de Thiès de 1996 à 2013 (ANACIM, 2013).

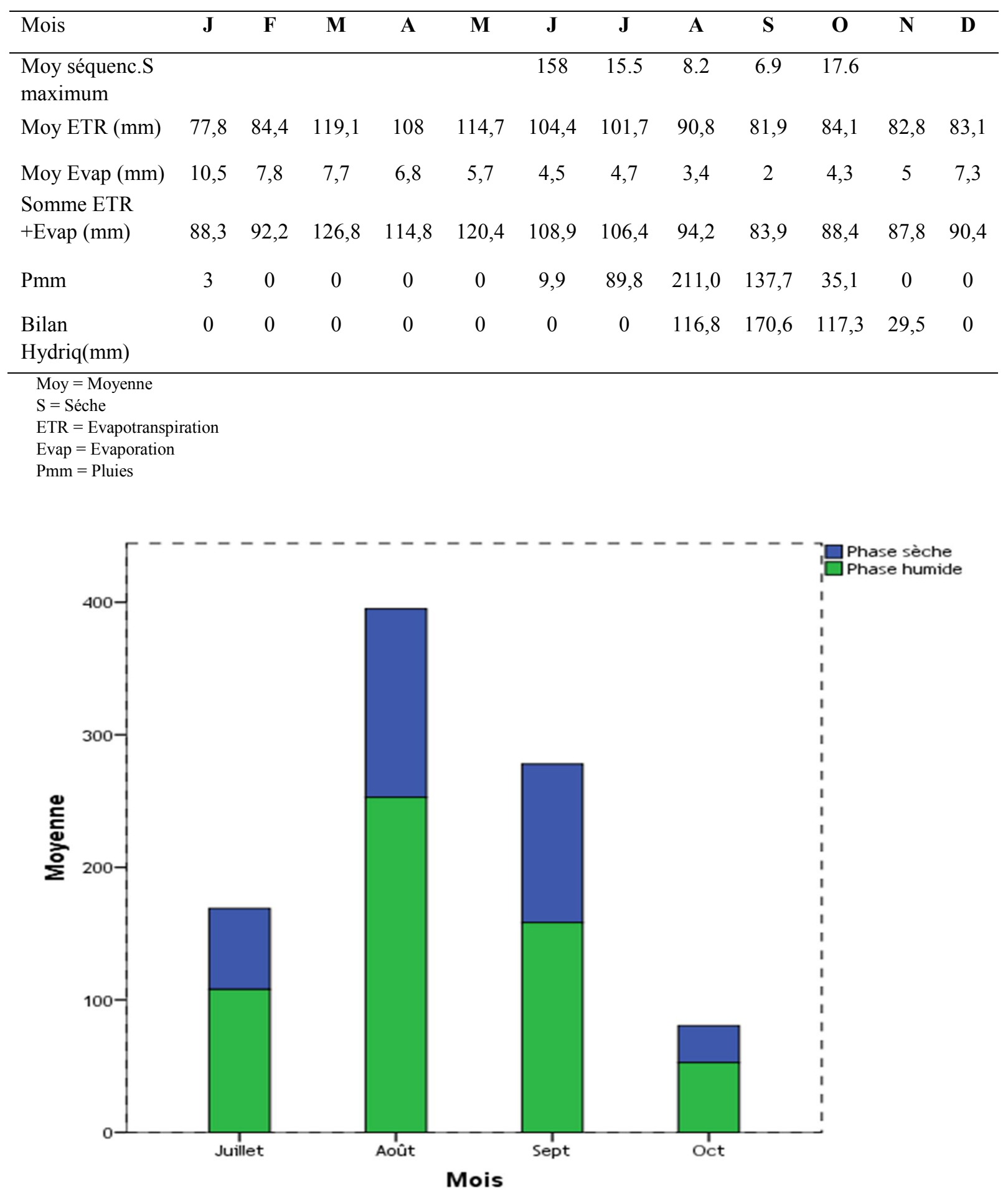

Figure 3 : Hausse de la pluviométrie $(\mathrm{mm})$ entre la phase sèche et la phase humide de la série 19962013 (ANACIM, 2013). 
Tableau 6 : Fréquences des épisodes secs de la phase sèche et de la phase humide de 1996 à 2013 (ANACIM, 2013).

\begin{tabular}{|c|c|c|c|c|c|c|c|c|c|c|c|}
\hline Années S & 1996 & 1997 & 1998 & 2002 & 2003 & 2004 & 2006 & - & - & - & - \\
\hline Fréq.Séq.S & 19 & 17 & 14 & 16 & 15 & 17 & 20 & - & - & - & - \\
\hline Années H & 1999 & 2000 & 2001 & 2005 & 2007 & 2008 & 2009 & 2010 & 2011 & 2012 & 2013 \\
\hline Fréq.Séq.S & 22 & 19 & 18 & 18 & 12 & 24 & 20 & 17 & 16 & 22 & 20 \\
\hline $\begin{array}{l}\text { Fréq = Fréquen } \\
\text { Séq = Séquence } \\
\mathrm{H}=\text { Humide } \\
\mathrm{S}=\text { Sèche }\end{array}$ & & & & & & & & & & & \\
\hline
\end{tabular}

Tableau 7 : Rentabilité des techniques de culture des exploitants du bassin.

\begin{tabular}{lcc}
\hline & \multicolumn{3}{c}{ Techniques de culture } \\
\cline { 2 - 3 } & Effectifs & $\%$ \\
\hline Insatisfait & 52 & 20,8 \\
Satisfait & 186 & 74,4 \\
Très satisfait & 12 & 4,8 \\
Total & 250 & 100 \\
Système manquant & 133 & 34,7 \\
\hline
\end{tabular}

\section{DISCUSSION}

Méthodes de lutte contre l'érosion des sols dans le bassin de la région de Thiès

Concernant les résultats sur l'efficacité des stratégies de lutte contre l'érosion instituées par les exploitants agricoles, on voit qu'ils sont conforment aux travaux de Ciss (2012) et Ndao (2013) qui estiment que les méthodes utilisées pour délimiter les champs, protéger le maraîchage et l'arboriculture fruitière mais aussi pour lutter contre l'érosion des sols ont largement contribué à la conservation et à la restauration des sols en ralentissant le ruissellement des eaux de pluie et en réduisant l'érosion éolienne. Les plantations de bois et les mesures de lutte contre le déboisement dans la région de Thiès ont permis de restaurer les sols dégradés et de les protéger contre les agents érosifs (ANSD, 2013; DRDR, 2013). Aussi, les travaux de Bazongo et al. (2015) et de Traoré et al. (2012) montrent que les plantations de Jatropha au Burkina Faso et au Niger ont été d'un grand apport pour la récupération et la protection des sols contre la dégradation et l'amélioration du niveau de la sécurité alimentaire. Les travaux de Ndiaye et al., (2012) révèlent que plusieurs stratégies (reboisement, phosphatage, mise en défends, etc.) ont été mises en œuvre pour la reconstruction des écosystèmes des Niayes.

Efficience des moyens de lutte mises en œuvre face aux difficultés hydriques

Plusieurs études telles que les travaux de Yanon et Ndiaye (2013), Sarr et al. (2010), 
et Ndong (2003) ont montré que le Sahel est confronté à un stress hydrique à l'instar des observations et des interrogations menées à l'égard des populations locales du bassin de la région de Thiès et que les solutions instaurées dans ce cadre n'ont pas atteints leur objectif. Les travaux de Sow (2011) montrent que les solutions entreprises dans le bassin du marigot d'Aga-Foua-Djilas et dans le Sahel sénégalais ont abouti à des échecs.

\section{Efficience des variétés agricoles à haut rendement}

Les résultats sur l'efficience des variétés à haut rendement (nouvelles variétés) issues des enquêtes de terrain tendent dans le même sens que ceux de l'Agence Nationale de Crédit Agricole et Rurale (ANCAR, 2014) sur les projets DGE et ERA portant sur la diffusion, la multiplication, l'usage de nouvelles variétés de mil et sorgho dans le bassin arachidier du Sénégal, l'intensification et la gestion de la production du mil dans des zones de Thiès, Louga et Nioro qui ont connu une réussite.

Effets de l'amélioration des conditions climatiques sur les rendements des exploitants

Les rendements et les productions élevés induits par l'amélioration des conditions climatiques récentes dans la présente étude sont soutenus par les travaux de l'ANSD (2013) et la DRDR (2013) sur la région de Thiès qui montrent que les productions agricoles annuelles sur la période de référence (1996-2013) sont plus fortes durant les dernières années avec un maximum recensé en 2008 (958673 Tonnes).

\section{Techniques de culture et leur efficience}

Les résultats sur la satisfaction des techniques de culture cadrent avec la satisfaction issue des projets de l'ANCAR (2014) et de la Fédération des Organisations Non-Gouvernementales du Sénégal (FONGS, 2015) où les producteurs locaux sont formés à de nouvelles pratiques et techniques de culture aboutissant à ces résultats satisfaisants.

\section{Conclusion}

L'agriculture dans le bassin de la région de Thiès est une activité confrontée à plusieurs contraintes dont l'insuffisance et la difficulté d'accès aux intrants agricoles de qualité, la vétusté et la difficulté de renouvellement du matériel agricole, les difficultés d'accès aux crédits agricoles. Les observations et les études de terrain ont montré que ces aspects influent sur les rendements et les revenus des exploitants. La réflexion a abouti à une diversité de solutions qui ont été mises en place sur l'espace du bassin de la région de Thiès au profit des exploitants agricoles. L'objectif était d'évaluer la rentabilité de ces actions mises sur pied pour le redressement et l'épanouissement de l'agriculture. Les résultats ont montré que les stratégies adoptées ont atteint leurs objectifs. Les méthodes de lutte ont été rentables pour la plupart à l'exception des moyens d'approvisionnement en eau agricole. L'efficacité de ces stratégies qui confère aux ménages agricoles des retombées économiques agricoles non négligeables explique le nombre plus élevé de ménages agricoles dans le bassin de Thiès comparé aux autres régions du Sénégal. Il advient comme perspective de mettre en place un système de crédit permanent pour une multiplication, une amélioration, une extension de ces stratégies d'adaptation dans tout le bassin de la région de Thiès mais surtout pour une maîtrise de l'eau qui pose le plus de problème dans les 
espaces ruraux et dans le bassin de la région de Thiès en particulier.

\section{CONFLIT D'INTERETS}

Les auteurs déclarent ne pas avoir de conflit d'intérêt pour cet article.

\section{CONTRIBUTIONS DES AUTEURS}

SD a collecté les données, les a analysées et rédigé le manuscrit. AN et HBN ont supervisé le travail.

\section{REMERCIEMENTS}

Nos gratitudes vont l'endroit les étudiants qui ont collecté les données de terrain et Monsieur NDIACK de la DAPSA qui nous a fourni les données agronomiques.

\section{REFERENCES}

Allen RG. 2010. Penman Monteith Evapotranspiration Calculations: Reference ET and Crop Coefficients, Univ. Idaho. Kimberly, p. 124. DOI: http://ccc.atmos.colostate.edu/ET_Works hop/pdf/2_Allen.pdf.

ANCAR. 2014. Rapport annuel d'activités de l'Agence Nationale du Conseil Agricole et Rural/Direction zone Niayes, Sénégal/Thiès, p. 75.

ANSD. 2013. Situation économique et sociale de la région de Thiès, Sénégal/MEFP/ANSD/SRSDT, Rapport d'activités, p. 75, [En ligne] URL: http://www.ansd.sn/index.php?option=co $\mathrm{m} \_$regions\&view $=$region $\&$ layout $=$ ses $\& \mathrm{i}$ $\mathrm{d}=5$. Consulté le 16 Juillet 2016 .

ANSD. 2010. Situation économique et sociale de la région de Thiès, Sénégal/MEFP/ANSD/SRSDT, Rapport d'activités, p. 90. [En ligne] URL : http://www.ansd.sn/index.php?option=co $\mathrm{m} \_$regions\&view $=$region $\&$ layout $=$ ses $\& \mathrm{i}$ $\mathrm{d}=5$. Consulté le 13 Juin 2015.
Bazongo P, Traoré K, Traoré O, Yélémou B, Sanon KB, Kaboré S, Hien V, Nacro HB. 2015. Influence des Haies de Jatropha sur le rendement d'une culture de sorgho (Sorghum vulgare) dans la zone Ouest du Burkina Faso : cas du terroir de Torokro, Int. J. Biol. Chem. Sci., 9(6): 2595-2607. DOI : http://www.ifg-dg.org.

Ciss A. 2012. La problématique de la conservation des sols dans la Communauté Rurale de Ngnith (Département de Dagana), Mémoire de Master II, UCAD/FLSH/Géographie, p. 109. DOI : http://bibnum.ucad.sn/ viewer.php?c=mmoires \&d=MemL-7328

Diallo S. 2012. Impacts socio-économiques et environnementaux de la dynamique des surfaces cultivées dans la Communauté Rurale de Fandène, Mémoire de Master II, UCAD/FLSH/Géographie, p.120.

DRDR. 2013. Rapport hebdomadaire de suivi de la campagne agricole, Sénégal/MAER/DRDR, p. 11.

FONGS. 2015. Rapport technique et financier $\mathrm{du} \quad 1^{\mathrm{er}} \quad$ semestre, $\quad$ p. 24. http://www.fongs.sn.

GIEC. 2007. Changement Climatique: Impacts, Adaptation et Vulnérabilité. Contribution du Groupe de Travail II au Quatrième Rapport d'Evaluation $d u$ Groupe Intergouvernemental sur l'Evolution du Climat, Annexe I, Parry ML, Canziani OF, Palutikof JP, van der Linden PJ, Hanson CE (Eds). Cambridge University Press: Cambridge, UK; 976.

Ndao AM. 2013. Stratégies de lutte contre la dégradation des sols le long de la vallée du Saloum dans la CR de Ribot Escale (Département de Koungheul), Mémoire de Master II, UCAD/FLSH/Géographie, p. 103. DOI : http://bibnum.ucad.sn/ viewer.php?c=mmoires\&d=meml_ 7840 . 
Ndiaye O, Diallo A, Matty F, Thiaw A, Fall RD, Guissé A. 2012. Caractérisation des sols de la zone des Niayes de Pikine et de Saint-Louis (Sénégal). Int. J. Biol. Chem. Sci., 6(1): 519-528. DOI : https://www.researchgate.net/publication 1272448195_Caracterisation_des_sols_d e_la_zone_des_Niayes_de_Pikine_et_de _Saint_Louis_Senegal.

Ndong JB. 2003. L'évolution climatique récente dans la région de Dakar, Annales/FLSH, n³3 : 91-108. DOI : http://bibnum.ucad.sn/viewer.php?c=arti cles\&d=ndong_jean_baptiste_1.

Owusu K, Klutse NAB. 2013. Simulation of the Rainfall Regime over Ghana from CORDEX, International Journal of Geosciences, 4: 785-791. DOI: http://file.scirp.org/pdf/IJG_2013062114 340225.pdf.

Sarr B, Ali B, Amani A, Ouaga HD, Garba I, Traoré S. 2010. Le sahel face aux changements climatiques: Enjeux pour un développement durable, bulletin mensuel, CILSS, numéro spécial, document PDF, $42 \quad \mathrm{p}$. http://www.cilss.bf/fondsitalie/download /down/specialChC.pdf. Consulté : le 07 Mai 2016.

Sow AA. 2011. Eau et économie rurale dans le bassin du marigot d'Aga-Foua-Djilas, Annales FLSH/n $/ n^{\circ}$ /1/B : 18-40. DOI : http://bibnum.ucad.sn/viewer.php?c=arti cles\&d=sow_amadou_abdoul.

Traoré M, Nacro HB, Tabo R, Nikiema A, Ousmane H. 2012. Potential for agronomical enhancement of millet yield via Jatropha curcas oilcake fertilizer amendment using placed application technique. Int. J. Biol. Chem. Sci., 6(2): 808-819.

Yanon G, Ndiaye A. 2013. Diminution observée des ressources en eau, une conséquence de la variabilité climatique? Etude basée sur une approche participative à Bambey (Sénégal), Géo-Eco-Trop., 37(1): 149156. DOI : http://accfp.org/docs/ library/journals/6\%20pub_371_11.pdf. 